STUDIA ROMANICA POSNANIENSIA

UAM Vol. 41/3 Poznań 2014

\title{
MIECZYSŁAW GAJOS
}

migaj@wp.pl

Université de Łódź

\section{LE BRUITAGE EN TANT QUE DECLENCHEUR DE LA CRÉATIVITÉ ET DE LA PAROLE EN CLASSE DE LANGUE}

\begin{abstract}
A bstract. Mieczysław Gajos, Le bruitage en tant que declencheur de la créativité et de la parole en classe de langue [Sound images as elements enhancing creativity and the speaking skill in a foreign language], Studia Romanica Posnaniensia, Adam Mickiewicz University Press, Poznań, vol. XLI/3: 2014, pp. 33-45. ISBN 978-83-232-2759-5. ISSN 0137-2475. eISSN 2084-4158. DOI: 10.7169/strop2014.413.003
\end{abstract}

To develop communicative competence in L2 does the learner need to have the knowledge of linguistic resources, but also of other factors shaping the situational context in which the act of communication is taking place. The elements bearing directly on efficiency on information exchange comprise all kinds of noises and sound images which make up a significant part of the broadly conceived communicative context. The present paper aims to raise the question of how the use of sound images in the process of foreign language learning/teaching should be made. Among other issues, it addresses the usefulness of various sounds and noises in stimulating learners' imagination, creativity and skills, as well as in enhancing spoken output in L2.

Keywords: sound images, noises, creativity, imagination, speaking skill

Le développement d'une compétence communicative en langue impose la prise en compte non seulement des composantes langagières d'une situation de communication mais aussi de celles du contexte situationnel au sens large du mot. L'importance de la mise en situation contextuelle des actes de parole a été largement développée dans les travaux du Conseil de l'Europe à l'occasion de l'élaboration d'un système européen d'unités capitalisables pour l'enseignement et l'apprentissage des langues étrangères. Connu sous le nom d'Un Niveau-Seuil, il a servi d'un document de référence de base pour l'élaboration des programmes et du matériel pédagogique pour l'enseignement et l'apprentissage des langues vivantes en Europe conformément à la méthodologie de l'approche communicative. (Porcher, 1979).

Le rôle des différents aspects du contexte qui déterminent l'utilisation de la langue à des fins communicatives a été minutieusement décrit dans un autre document du Conseil de l'Europe : Un cadre européen commun de référence pour les langues (CECRL). 
Les auteurs de ce document précisent que " le contexte renvoie à la multitude des événements et des paramètres de la situation (physiques et autres), propres à la personne mais aussi extérieurs à elle, dans laquelle s'inscrivent les actes de communication » (CECRL, $2001:$ 15).

Parmi les contraintes imposées par le cadre extérieur dans lequel se déroule un acte de communication orale et qui influencent directement l'efficacité de cet acte, il faudrait mentionner:

- clarté de la prononciation (diction),

- bruit ambiant (trains, avions, etc.),

- interférences (rue bondée, marchés, cafés, soirées, discothèques, etc.),

- distorsions (mauvaises lignes téléphoniques, réception par radio, systèmes de sonorisation),

- conditions météorologiques (vent, froid extrême, etc.) (CECRL, $2001: 42$ ).

Il faudrait que dès le début de leur apprentissage d'une langue étrangère, les élèves se familiarisent non seulement avec les moyens langagiers dont ils auront besoin pour concevoir des messages qui leur permettront de réaliser toutes sortes de tâches communicatives mais il serait aussi souhaitable qu'ils puissent prendre conscience des autres facteurs extra-linguistiques qui entrent en jeu pour comprendre et s'exprimer correctement et efficacement dans toute situation de communication.

Dans cet article on s'interrogera sur le rôle des bruitages dans le contexte de la didactique des langues vivantes. Notre étude portera en particulier sur l'exploitation pédagogique des images acoustiques dans une classe de FLE avec pour objectif le développement de la créativité et de l'expression orale des apprenants.

On partira d'une présentation de l'évolution du concept de créativité, pour décrire ensuite la place et les fonctions des bruitages dans l'apprentissage et l'enseignement des langues dans le contexte scolaire. On verra comment les images acoustiques qui remplacent les images visuelles peuvent éveiller et augmenter l'intérêt des élèves, les inciter à faire travailler leur imagination tout en leur donnant l'occasion de s'exprimer librement en langue étrangère. On aboutira enfin à la présentation du matériel pédagogique conçu à partir des bruitages qui ont pour objectif de faire développer les capacités communicatives des apprenants et leur créativité langagière. On présentera quelques activités et tâches exploitant les différentes sources de bruits qui peuvent être utilisées en classe de FLE: d'un bruit isolé à une série de bruits.

\section{LA CRÉATIVITÉ ET L'APPRENTISSAGE D’UNE LANGUE ÉTRANGÈRE}

Au sens courant du mot, la créativité est un pouvoir de création, d'invention. (Ray-Debove 1993: 505). Etre créatif c'est donc être capable de créer et d'inventer. La créativité de l'homme peut se manifester dans plusieurs domaines de vie, il nous 
serait impossible d'en énumérer tous. L'exemple le plus simple et le plus significatif c'est celui du monde de la découverte ou encore celui de l'art.

La créativité dans le contexte d'une classe de langue devrait êre examinée de quelques points de vue: linguistique (la langue étant un objet d'enseignement et d'apprentissage), psychologique (pour mieux comprendre la compléxité des phénomènes et des mécanismes qui gèrent l'esprit de l'apprenant) et didactique (étant donné l'environnement dans lequel un apprenant acquiert une langue étrangère).

La créativité a été largement étudiée par les représentants de chaque domaine respectif et à partir des années soixante-dix du $\mathrm{XX}^{\mathrm{e}}$ siècle de nombreux travaux ont été publiés dans le monde entier pour faire approcher les différentes conceptions théoriques de cette notion. (Beaudot, 1973; Rouquette, 1973; Demory 1974; Szmidt, 2010; Nęcka, 2012).

Pour ce qui est de la linguistique, il faut avant tout citer les travaux de Noam Chomsky pour qui la créativité est une faculté fondamentale de notre langage (Chomsky, 1975). D'après sa théorie, grâce aux règles récursives qui existent dans chaque système linguistique, un sujet parlant peut former un nombre infini d'énoncés nouveaux avec un nombre fixe d'éléments qu'offre une langue.

Il faudrait aussi rappeler la définition de créativité sémantique proposée par Strawson en 1970 pour qui la créativité est: « une propriété inhérente aux langues naturelles, mais liée à l'acte d'énonciation qui permet au locuteur l'exploitation de ressources sémantiques considérables, soit pour modaliser, nuancer, infléchir le sens linguistique d'un énoncé, soit pour choisir la manière de communiquer du sens » (cité par: Caré, Debyser, 1978 : 118).

Quant à la psychologie, la créativité est indentifiée à une aptitude d'intelligence qui peut être mesurée dans les tests de Q.I. Coste et Galisson énumèrent quatre définitions différentes adoptées par les psychologues :

- « capacité d'adaptation à des circonstances et à des conditions naturelles imprévues ;

- facilité à tirer parti de l'expérience et à acquérir des connaissances en leur trouvant des modalités d'emplois ou des contextes d'application non déjà entrevus ;

- pouvoir de renouvellement dans un domaine d'activité donné ;

- éveil et mise en forme de dispositions naturelles pour l'accomplissement d'un acte créateur » (Coste, Galisson, $1976: 135)$.

Les travaux empiriques des psychologues sur les caractéristiques de la créativité de l'esprit humain, parmi lesquelles : la fuidité, la flexibilité et l'originalité ont donné naissance à des techniques qui avaient pour objectif de faire stimuler l'imagination constructive des individus. Le brain storming ou la simulation globale sont les techniques les plus connues qui ont été appliquées dans plusieurs domaines professionnels y compris dans le domaine de l'éducation.

Les pédagogues, quant à eux, aperçoivent dans la créativité la capacité d'imaginer et d'inventer. Un apprenant créatif c'est celui qui a de l'initiative, qui a des idées, qui 
cherche à être original par ce qu'il fait et comment il le fait. Dans le contexte scolaire, les activités et opérations intellectuelles qu'il doit exécuter le mènent à la découverte, à la production et à l'expression.

En reprenant les idées citées ci-dessus proposées par les linguistes, psychologues et pédagogues, Daniel Coste et Robert Galisson admettent que dans le contexte de la didactique des langues la créativité est une « aptitdue de l'élève à inventer et à créer des énoncés dans des cadres thématiques ou situationnels qu'il découvre lui-même » (Coste, Galisson, 1976 : 136).

Michel Sagaz souligne de son côté que la créativité est un processus qui «provoque l'apprentissage qui génère la motivation ». «Susciter la créativité des apprenants n'implique pas pour l'enseignant de travailler sur l'objet de l'apprentissage mais bien de travailler sur les apprenants. En ce sens, nous pouvons dire que la créativité est un processus, et si on a mis en avant la question du « qui apprend? ,celle du « comment on apprend ?" lui est donc directement liée » (Sagaz, 2006 : 46).

En traduisant les différents concepts de la créativité proposés par la linguistique, la psychologie et la pédagogie, et en les faisant transférer dans le contexte d'une classe de langue, Jean-Pierre Robert remarque que la créativité de l'apprenant (au sens où l'entend la linguistique) ne peut être stimulée que par une attitude ouverte de l'enseignant à toute production langagière de l'élève. D'après le même auteur : « la classe de langue est un lieu privilégié pour cultiver la créativité naturelle d'un apprenant (au sens où l'entend la psychologie). Les enseignants et les concepteurs de manuels proposent, soit des exercices isolés, soit de véritables projets destinés à la développer au cours des séances, appelées parfois à juste titre ateliers, puisqu'elles visent une création » (Robert, $2002: 45)$.

Dans la didactique du français langue étrangère, la notion de créativité a été proposée et développée par Jean-Marc Caré et Francis Debyser qui par leur ouvrage Jeu, langage et créativité. Les jeux dans la classe de français ont fait approcher aux professeurs de FLE quelques pistes méthodologiques concernant le développement de la créativité chez les élèves qui apprennent des langues vivantes étrangères en situation scolaire.

Ils ont entre autres défini et décrit d'une façon détaillée les conditions favorables à la créativité dans la classe, et surtout dans la classe de langue :

- le travail en groupe : favorise les échanges, la production et diversification des idées ;

- le climat du groupe : gai, détendu ;

- l'absence de hiérarchie : les inégalités de statut empêchent et inhibent l'inventivité ;

- la levée des censures : l'esprit critique, la routine et le conformisme brident l'imagination ;

- la mise en commun : la mise en commun de l'inventivité dans un groupe abat le cloisonnement et favorise le changement d'idées et la mise au point ; 
- créativité et régression : une certaine régression est nécessaire pour devenir créatif ; un retour en arrière, une régression permet au sujet de se retrouver lui-même pour devenir ou redevenir novateur ou créateur (Caré, Debyser, 1978 : 120-121).

Afin de développer la créativité des apprenants en classe de langue, l'enseignant devrait donc faire tout son possible pour activer l'imagination et l'inventivité des élèves. Pour cela, il serait souhaitable qu'il propose au public d'apprenants des tâches et des activités qui font réfléchir tout en favorisant leur esprit critique. Seules les activités où les apprenants auront à chercher, à découvrir, à formuler des hypothèses, à les discuter en commun, à échanger leurs opinions, à les vérifier et à les exposer permettent de développer la créativité en classe de FLE. Ce genre d'activités à caractère créatif motive les élèves et les encourage à participer activement à une leçon. En appliquant les techniques qui visent au développement de la créativité des apprenants, le professeur de langue les entraîne systématiquement à s'exprimer librement et spontanément à l'oral et à l'écrit. Le travail en classe ne devrait pas se dérouler dans une ambiance contraignante où le professeur monopolise la parole, les savoir et les savoir-faire. Il faudrait que le professeur sache stimuler ses élèves au travail, que le cours se réalise dans une ambiance de coopération et de franchise sans crainte ou peur d'être sanctionné pour avoir commis une faute ou pour avoir exprimé une idée qui ne correspond pas à celle de l'enseignant ou à d'autres élèves. Il faut faire apprendre à partager, à discuter et à accepter les opinions des autres. Si l'on veut développer la créativité dans une classe de langue au sens linguistique, psychologique et didactique, l'enseignant et les élèves doivent rédefinir leurs rôles dans le processus didactique afin de créér des conditions favorables au changement d'idées et d'énoncés verbaux. "Susciter la créativité chez les apprenants, c'est leur donner les moyens d'agir face à une situation problématique donnée. Pour l'enseignant, reconnaître chez les apprenants leur créativité, c'est les rendre responsables de leur apprentissage » (Sagaz, $2006: 49)$.

Le professeur en tant que facilitateur d'apprentissage et les élèves en tant qu'utlisateurs conscients d'un autre mode de commnication langagière qui partagent la responsabilité de leur apprenstissage peuvent donc facilement arriver à briser la structure rigide des rôles joués dans une classe de langue et accepter des procédures qui visent au développement de la créativité.

\section{LE BRUITAGE ET LE DÉVÉLOPPEMENT DE L’IMAGINATION ET DE LA CRÉATIVITÉ EN CLASSE DE LANGUE}

Le monde des bruits nous accompagne dans les différentes situations de notre vie quotidienne. Les stimuli auditifs auxquels nous sommes exposés fournissent des informations sur le monde qui nous entoure, provoquent les activités langagières, dirigent nos activités extra-linguistiques, etc. 
Dans les théories de l'information et de communication, le bruit est un élément perturbateur qui gêne la transmission d'un message. Le bruit apparait entre autres dans le modèle mathématique de communication linguistique élaboré par Shannon et Weaver (Shannon, Weaver, 1962). «L'objectif de Shannon est de dessiner le cadre mathématique à l'intérieur duquel il est possible de quantifier le coût d'un message, d'une communication entre les deux pôles de ce système, en présence des perturbations aléatoires, dites „,bruit” indésirables parce qu'empêchant l',,isomporphisme”, la pleine correspondance entre les deux pôles » (Mattelart, 2004 : 31).

Les parasitages qui déforment le message ou rendent son passage de l'émetteur au réceptuer impossible sont donc à éliminer ou au moins à réduire. Il est évident que le sifflement d'un train qui entre en gare et qui couvre l'annonce prononcée par le haut-parleur peut être considéré comme un effet perturbateur qui trouble la communication. Mais le même sifflement d'un train peut consituer un élément ambiant nous annonçant par exemple l'entrée en gare ou le danger au passage à niveau.

Nous allons abandonner les bruits au sens linguistiques et allons nous concentrer sur l'aspect ambiant des bruits qui aident les interlocuteurs à saisir le contexte dans lequel se déroule la communication. Nous allons aussi axer notre attention sur les images acoustiques qui peuvent remplacer la réalité concrète ou les images visuelles tout en apportant aux interlocuteurs les informations qui mènent à la découverte du sens de message. Les images acoustiques développent l'imagination des apprenants, leur permettent de créer leur propre vision du monde sans imposer celle qui est inscrite et sugérée sur les photos, tableaux, dessins ou autres formes d'images visuelles. Par exemple, le sifflement d'un train cité ci-dessus permet d'identifier le moyen de transport mais n'apporte aucune information supplémentaire : train de marchandises ou train de voyageurs? Train en arrivée ou train en partance? Train avec ou sans wagons ? Combien de wagons ? Y-a-t-il un wagon restaurant? Y-a-t-il un wagon de poste ? Y-a-t-il un wagon-lits ? Est-ce un omnibus, express, ou rapide?

Les images acoustiques renvoient donc à l'inventivité et à la créativité de l'individu. Elles peuvent rendre la transmisson des messages verbaux plus efficaces et faciliter aux interlocuteurs l'accès au sens des messages linguistiques. En plus, les images acoustiques permettent de développer et d'explorer la mémoire de l'oreille. Il est donc possible d'exploiter en classe de langue les images sonores stockées antérieurement dans la mémoire de l'élève, par exemple celles découvertes et enregistrées lors de son développement cognitif accompagné de l'acquisition de sa langue maternelle.

Les bruits ambiants sont souvent utilisés dans le bruitage qui accompagne une trame sonore des productions multimedia. On retrouve les images acoustiques dans les pièces radiophoniques, dans les livres audio, à la télé, au cinéma, au théâtre mais aussi dans les jeux vidéo ou ceux accessibles sur Internet. Dans le monde des media la notion de bruitage est résérvé aux bruits fabriqués d'une façon artificielle. Il est réalisé en studio d'enregistrement avec des moyens et accessoires qui permettent de recréer le son voulu aussi fidèlement que possible. Bien évidemment, on utilise aussi les sons 
authentiques pré-enregistrés et stockés dans les banques des sons dits sonothèques. Sur internet, tout enseignant peut facilement trouver les bruitages et les sons d'ambiance à télécharger gratuitement (voir quelques adresses dans la bibliographie). Les sonothèques fournissent des images acoustiques classées par thème avec lesquelles on peut concevoir toutes sortes d'activités didactiques pour une classe de FLE.

Un simple bruit, une simple image acoustique est capable de provoquer en classe des réactions verbales des élèves. L'identification du son n'étant pas aussi évidente que celle de l'image visuelle permet de travailler avec les apprenants les expressions qui donnent la possibilité d'exprimer leurs opinions, de faire des hypothèses et de les vérifier.

L'enregistrement des pas dans le couloir fait penser à une personne. Mais qui estelle ? Est-ce un homme ou une femme ? Est-elle jeune ou vieille ? Comment est-elle ? Où va-telle? Comment est-elle habillée ? etc.

Les élèves auront à utiliser non seulement les expressions qui leur permettent d'identifier, de désigner ou de caractériser la personne à qui appartiennent les pas :

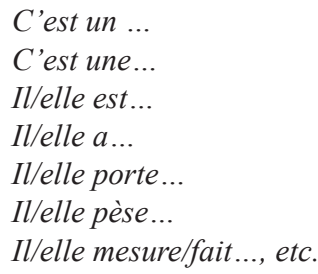

mais aussi celles grâce auxquelles il est possible d'exprimer l'opinion, la certitude, le doute, l'accord ou le désaccord:

D'après moi...

Selon moi...

Quant à moi...

A mon avis...

Pour moi...

Je pense que...

Je crois que...

Il me semble que...

J'ai l'mpression que...

Je suis sûre que...

Il est clair que...

Il est évident que...

Je suis convaincu que...

Je suis persuadé que...

Je suis d'accord que...

Bien entendu...

Vous avez raison... / tu as raison...

Je suis tout à fait de votre avis...

Je ne suis pas d'accord que...

Je ne suis pas tout à fait d'accord que... 
Je ne crois pas que...

Je ne pense pas que...

Ça m'étonnerait que... etc.

Comme nous le voyons à travers ce simple exemple, l'image acoustique qui renvoie à l'imagination de l'élève et qui peut être interprétée de plusieurs façons donne aux apprenants beaucoup plus de possibilités de prise de parole qu'une image visuelle. D'après nos observations en classes de FLE, elle sert facilement de déclencheur de la parole tout en permettant de développer l'imagination et la créativité de l'élève. Dans la suite de cet article nous présentons les grandes lignes du projet pédagogique Ouvrez-vos oreilles conçu pour l'enseignement et l'apprentissage du FLE au public scolaire polonais. Cette présentation générale du projet sera suivie de quelques activités qui exploitent les images acoustiques tout en permettant aux élèves de réaliser un certain nombre d'objectifs communicatifs et/ou linguistiques.

\section{OUVREZ VOS OREILLES! OU COMMENT EXPLOITER LES IMAGES SONORES EN CLASSE DE FLE}

Le projet pédagogique Ouvrez vos oreilles a été conçu pour le public scolaire polonais jeunes adolescents 11-12 ans, niveaux A-1 / A2) afin de développer la créativité langagière de l'élève dans le contexte communicatif. Pour atteindre cet objectif fondamental, nous nous sommes référés à une situation de simulation globale où l'on fait produire des échanges verbaux à partir du matériel pédagogique composé d'un livret d'activités et des images acoustiques pré-enregistrées.

Pour notre projet pédagogique nous avons défini deux grands groupes d'objectifs : linguistiques et communicatifs, parmi lesquels :

- savoir écouter et reconnaître des messages sonores non-linguistiques qui remplacent la communication verbale ou qui l'accompagnent ;

- développer l'expression orale de l'élève en langue étrangère à partir de différentes images acoustiques ;

- faire remarquer le rôle du non-verbal dans un acte de communication ;

- savoir formuler des hypothèses sur le sens du message ;

- savoir exprimer son opinion et prendre position dans une discussion;

- faire connaître le lexique concernant les centres d'intérêts très diversifiés de l'élève ;

- faire fixer des structures morphosyntaxiques de base qui permettent de réaliser en langue un certain nombre d'actes de parole.

Dès le début de l'élaboration de notre projet, nous avons cherché à trouver un cadre de communication simulée qui répondrait aux centres d'intérêt du public de jeunes apprenants et dans lequel les élèves prendraient l'initiative de communiquer. Tout au long de la réalisation du projet, les apprenants sont invités à réaliser plusieurs 
tâches qui leur donnent l'occasion de pratiquer une langue étrangère dans différentes situations de communication quotidienne.

Ce projet est donc entièrement centré sur l'apprenant, sur ses besoins communicatifs et langagiers. Il lui assure une position privilégiée dans le contexte didactique. On admet qu'il sait plus que les autres sujets du projet. L'élève est en permanence sollicité pour partager son savoir et ses savoir-faire en langue étrangère avec son instituteur et avec d'autres membres du groupe-classe. Grâce à cette approche, on observe le renversement des rôles : maître-élève. Contrairement à la situations scolaire où les rôles d'enseignant et d'apprenant sont définis d'office, dans notre projet de simulation globale, ils varient en fonction de la situation et du contexte dans lequel se trouve à un moment donné l'enfant.

Quel est donc ce cadre qui nous a permis de placer l'apprenant dans une situation d'apprentissage actif? Il s'agit d'un extra-terrestre qui cherche à étudier les sons de la Terre. Sa planète s'appelle La Sonolande. C'est une toute petite planète qui se trouve quelque part dans l'univers. Son roi, Tenorus VII a une seule mais grande passion : collectionner les sons venant des autres planètes. Dans l'un de ses laboratoires équipés en appareils d'enregistrement très sophistiqués, on vient de capter les sons d'une planète qui se trouve dans le système solaire. Cette planète s'appelle la Terrelande. Le roi décide d'y envoyer le professeur Trombolus, son plus grand ami et en même temps le meilleur chercheur dans le domaine du son. Il lui confie une mission spéciale : enregistrer et répertorier tous les sons de cette planète nouvellement découverte. Ce sympathique extra-terrestre enregistre donc tous les bruits de la Terrlande. Il y en a tant que le pauvre professeur Trombolus n'arrive plus à les gérer. Ce vieux scientifique est tellement gentil et sympatique qu'il faut l'aider. Qui va le faire ? Les élèves, bien sûr ! Et c'est là qu'une vraie aventure avec les images sonores et le français commence.

L'élève reçoit donc le matériel dont il devient l'un des co-auteurs. La conception pédagogique de son journal de bord (cahier d'activités) demande de l'élève d'être tout le temps actif, inventif et créatif. Il est en permanence sollicité pour résoudre des problèmes, réaliser des tâches, observer, inventer, analyser, proposer, s'exprimer, etc. Toutes les activités construites à partir de différentes sources de bruits et de sons font travailler le potentiel intellectuel, cognitif et émotif de l'élève. Le processus d'apprentissage devient une activité ludique, une simulation, une activité motivante tout en permettant à l'apprenant de développer ses compétences communicative et linguistique en langue étrangère.

Dans le tableau ci-dessous, nous présentons la structure du matériel utilisé en classe dans le cadre du projet Ouvrez vos oreilles. Chaque unité permet d'entraîner les apprenants à réaliser un autre acte de parole. L'élève a l'occasion de réutiliser un certain nombre de structures grammaticales dans le contexte de communication simulée. En apportant de l'aide au professeur Trombolus pour identifier, classer et décrire les sons de la Terrlande, l'apprenant découvre et mémorise le lexique lié aux différents centres d'intérêt. 


\begin{tabular}{|c|c|c|}
\hline Unités & Titre & Actes de parole \\
\hline 1. & Qu'est-ce que c'est? & $\begin{array}{l}\text { - demander l'identité d'un objet } \\
\text { - savoir nommer et décrire les objets } \\
\text { - exprimer son avis }\end{array}$ \\
\hline 2. & A quoi ça sert? & $\begin{array}{l}\text { - s'informer ou informer quelqu'un sur le } \\
\text { fonctionnement d'un outil, d'un appareil, } \\
\text { etc. } \\
\text { - donner des consignes, des ordres }\end{array}$ \\
\hline 3. & Qui est-ce? & $\begin{array}{l}\text { - indentifier quelqu'un } \\
\text { - présenter quelqu'un : dire son nom, } \\
\text { prénom, âge, sexe, origine, langues } \\
\text { parlées, profession, etc. } \\
\text { - demander de se présenter }\end{array}$ \\
\hline 4. & Les reconnaissez-vous? & $\begin{array}{l}\text { - savoir indentifier quelqu'un } \\
\text { - décrire quelqu'un } \\
\text { - caractériser quelqu'un } \\
\text { - faire des hypothèses sur l'identité de } \\
\text { quelqu'un } \\
\text { - exprimer le doute, la certitude, etc. }\end{array}$ \\
\hline 5. & Où es-tu? Où sont-ils? Quand? & $\begin{array}{l}\text { - localiser dans l'espace } \\
\text { - localiser dans le temps } \\
\text { - décrire un lieu }\end{array}$ \\
\hline 6. & Qu'est-ce qu'ils font? & $\begin{array}{l}\text { - savoir nommer les actions en cours } \\
\text { - savoir donner des ordres } \\
\text { - savoir décrire les actions }\end{array}$ \\
\hline 7 & $\begin{array}{l}\text { Comment sont-ils? Quels sentiments } \\
\text { expriment-ils? }\end{array}$ & $\begin{array}{l}\text { - décrire, exprimer les émotions } \\
\text { - porter un jugement }\end{array}$ \\
\hline 8. & Qu'est-ce qu'on dit quand on entend...? & $\begin{array}{l}\text { - savoir réagir en langue à des bruits } \\
\text { extralinguistiques }\end{array}$ \\
\hline 9. & Les petites histoires à partir des bruits & $\begin{array}{l}\text { - raconter une suite d'événements } \\
\text { - raconter en exprimant les relations } \\
\text { temporelles } \\
\text { - rapporter }\end{array}$ \\
\hline 10. & Savez-vous imiter les bruits? & - savoir imiter les bruits à la française \\
\hline
\end{tabular}

Pour chaque unité didactique, on a prévu le lexique et la grammaire indispensables pour réaliser efficacement et correctement les actes de parole pré-sélectionnés. À titre d'exemple, dans la première unité, les élèves apprennent le lexique concernant : les animaux, les moyens de transport, les instruments de musique, les phénomènes de la nature, les saisons de l'année et les objets divers.

Quant à la grammaire, les apprenants s'entraînent à utiliser les structures suivantes : 
- questions : Qu'est-ce que c'est? Comment ça s'appelle? Comment est-il/elle?

- c'est / ce sont + articles indéfinis / définis

- masculin / féminin, singulier / pluriel des substantifs et des adjectifs

- expressions : à mon avis, d'après moi, je pense que, etc.

Pour terminer la présentation du projet Ouvrez vos oreilles, regardons de près quelques activités ayant pour objectif de faire développer l'expression verbale des apprenants et leur créativité.

Activité 1.

Aidez le professeur Trombolus à trouver les noms des instruments de musique qu'il a enregistrés. Comment appelle-t-on les musiciens qui utilisent ces instruments dans un orchestre? A quels animaux pensez-vous quand vous entendez les sons des instruments enregistrés?

\begin{tabular}{|l|l|l|}
\hline Instruments de musique & Musiciens & Animaux \\
\hline & & \\
\hline & & \\
\hline & & \\
\hline
\end{tabular}

Activité 2.

Ecoutez quatre extraits de musique. Fermez vos yeux. A quelles saisons de l'année vous font-ils penser? Quels instruments de musique entendez-vous dans chaque extrait? (Enregistrement de „Les quatre saisons de l'année” d'Antonio Vivaldi).

Activité 3 .

Quels instruments de musique pourriez-vous utiliser pour faire imiter les bruits de la nature?

\begin{tabular}{|l|l|}
\hline \multicolumn{1}{|c|}{ Bruits de la nature } & Instruments de musique \\
\hline La pluie & \\
\hline Les vagues de la mer & \\
\hline Le tonnerre & \\
\hline Le vent & \\
\hline Le ruisseau & \\
\hline
\end{tabular}

Activité 4.

Fermez vos yeux, écoutez attentivement et dites où vous êtes? Décrivez ce que vous voyez.

(la forêt, la campagne, la plage, la ville, l'aéroport, la gare, l'usine, l'école, etc.). 
Activité 5.

Ecoutez les extraits de concerts enregistrés par le professeur Trombolus. Pourriezvous l'aider à nommer les styles de musique?

(la musique classique, le jazz, le disco, le pop, le rap, la musique électronique, le rock, le heavy metal).

Activité 6.

Qui sont-ils? Reconnaissez-vous leurs voix? Aidez le professeur à trouver les noms des chanteurs et acteurs français. Trouver sur le net les photos de ces artistes et ajoutez-les dans le journal de bord du professeur Trombolus.

Activité 7.

Ecoutez attentivement les voix de quelques personnages célèbres. Fermez les yeux. Comment les voyez-vous ? Comment sont-ils? Caractérisez-les d'après les traits de leur voix.

Activité 8.

Ecoutez une suite de bruits enregistrés par le professeur Trombolus. Devinez ce qui s'est passé. Racontez-le.

(la chouette qui hulhule, le loup qui hurle, la pluie et l'orage, les pas, le battement de cœur, le tambour, les chiens, les tirs de fusil, les bravos, les rires).

\section{CONCLUSION}

Dans notre projet Ouvrez vos oreilles nous avons remplacé les images visuelles par les images sonores tout en étant persuadés que ces dernières permettent de déclencher les énoncés verbaux plus authentiques du point de vue communicatif. D'après notre propre expérience et nos observations systématiques menées en classes de FLE où on a réalisé le projet Ouvrez vos oreilles, les bruits ambiants, les images acoustiques assurent l'acquistion plus naturelle et authentique des moyens linguistiques indispensables pour apprendre à exprimer son opinion, son accord, son désaccord, pour savoir contredire ou argumenter. Grâce au matériel pédagogique présenté ci-dessus, les élèves arrivent à développer leur expression en langue étrangère à l'oral et / ou à l'écrit. En partant des énoncés relativement simples, ils arrivent progressivement à construire des textes narratifs dans lesquels ils doivent mettre en jeu toutes leurs connaissances acquises antérieurement.

Les images sonores utilisées en tant que déclencheurs de la parole rendent les élèves attentifs, inventifs et créatifs tout au long du projet auquel ils participent en classe de FLE. 


\section{RÉFÉRENCES}

BEAudot, Alain (1973): Vers une pédagogie de la créativité. Paris : Les Éditions sociales françaises. CARe, Jean-Marc; Debyser, Francis (1978): Jeu, langage et créativité. Les jeux dans la classe de français. Paris : Hachette / Larousse.

Сномкку, Noam (1975): The logical structure of lingustic theory. New York : Plenum Press.

Clere, Stéphanie (2003): « L'utilisation des bruitages dans le développement de la compétence narrative ». In : Manfred Friedrich PrINZ (éd.), FFF - Frühes Fremdsprachenlernen Französisch II, Giessen : Gunter Narr Verlag Tübingen, 48-57.

Conseil de L'europe, (2001): Cadre européen commun de référence pour les langues, Paris : Les Éditions Didier.

Coste, Daniel; Galisson, Robert (1976): Dictionnaire de didactique des langues. Paris : Hachette.

Demory, Bernard (1974): La créativité en pratique. Paris : Chotard et Associés.

GaJos, Mieczysław (2003): « Du non-linguistique au communicatif». In : Manfred Friedrich PrINZ (éd.), FFF - Frühes Fremdsprachenlernen Französisch II, Giessen : Gunter Narr Verlag Tübingen, 41-47.

KARPETA-PEĆ, Beata (2008): Otwarty, aktywny, samodzielny ... alternatywne formy pracy : przewodnik dla nauczycieli języków obcych. Warszawa : Fraszka Edukacyjna.

Mattelart, Armand et Michèle (2004): Histoire des théories de la communication. Paris : La Découverte.

NĘCKA, Edward (2012): Trening twórczości. Gdańsk : Gdańskie Wydawnictwo Psychologiczne.

NęcKA, Edward (2012): Psychologia twórczości. Gdańsk : Gdańskie Wydawnictwo Psychologiczne.

PorCHER, Louis (1986): Adaptation de "Un niveau seuil » pour des contextes scolaires. Paris : Hatier.

RoBert, Jean-Pierre (2002): Dictionnaire pratique de didactique du FLE. Paris : Éditions OPHRYS.

SAgaz, Michel (2006) : « De la créativité à l'autonomie ». In : Małgorzata PamuŁa ; Anita Pytlarz (éd.), Diversité des recherches en langues-cultures. Synérgies Pologne, 3/2006, 43-52.

Rey-Debove, Josette; Rey, Alain (1993): Le Nouveau Petit Robert. Paris : Dictionnaires le Robert.

Rouquette, Michel-Louis (1973): La créativité. Paris : PUF, Que sais-je ?

SzmidT, Krzysztof J. (2005): Dydaktyka twórczości. Kraków : Wydawnictwo Impuls.

Szmid, Krzysztof J. (2010): ABC kreatywności. Warszawa : Difin.

Szmidt, Krzysztof J. (2012): Pedagogika twórczości. Gdańsk : Gdańskie Wydawnictwo Psychologiczne.

WeISs, François (1983) : Jeux et activités communicatives dans la classe de langue. Paris : Hachette.

\section{SOURCES ÉlectroniQues}

Pages web avec les images sonores

Source électronique 1 :

< http://www.universal-soundbank.com>. Consulté le 12 mai 2014.

Source électronique 2 :

$<$ http://lasonotheque.org/>. Consulté le 12 mai 2014. 